\title{
INTERVENCIÓN EN DOCENTES PARA EL DESARROLLO DE COMPETENCIAS PSICOMOTORAS EN NIÑOS A NIVEL PREESCOLAR
}

\author{
Pedro Erick Gastelum Acosta* \\ https://orcid.org/0000-0002-4776-4687 \\ Rigoberto Marín Uribe** \\ https://orcid.org/0000-0002-8672-4047 \\ Isabel Guzmán Ibarra*** \\ https://orcid.org/0000-0001-6075-5024 \\ José Aldo Hernández Murúa**** \\ https://orcid.org/0000-0002-9277-5799
}

RECIBIDO: Abril 2021 / ACEPTADO: Octubre 2021 / PUBLICADO: Enero 2022

\begin{abstract}
Como citar: Gastelum Acosta, Pedro; Marín Uribe, Rigoberto; Guzmán Ibarra, Isabel; Hernández Murúa, José. (2022). Intervención en docentes para el desarrollo de competencias psicomotoras en niños a nivel preescolar. Telos: revista de Estudios Interdisciplinarios en Ciencias Sociales, 24 (1), Venezuela. (Pp. 79-99).

DOI: www.doi.org/10.36390/telos 241.06
\end{abstract}

\section{RESUMEN}

El objetivo del estudio fue describir los resultados de la aplicación del programa educación preescolar apoyado del modelo M-DECA en los docentes para el desarrollo de las competencias psicomotoras en el campo formativo de desarrollo físico y salud en los niños. El estudio tuvo un enfoque cualitativo, investigación colaborativa, método investigación acción participante (IAP), participaron 6 docentes y un grupo de 47 niños y niñas de una institución de preescolar. Se utilizó el modelo M-DECA de Guzmán, Marín e Inciarte (2014), centrando en una práctica guiada de 7 actividades, que fueron desarrolladas en cinco fases integrales, cada participante cumplió varios roles, a quienes se les aplicaron instrumentos y técnicas cualitativas sistematizadas por el software ATLAS ti. Los hallazgos encontrados luego de la aplicación de la secuencia

\footnotetext{
* Doctor en Ciencias de la Cultura Física. Especialista en Educación física, formación docente y gestión educativa. Profesor investigador tiempo completo en la Universidad Autónoma de Sinaloa. Unidad regional centro, Culiacán, Sinaloa, México. Catedrático de las Facultades de Educación Física y Deporte y de Trabajo Social. Correo electrónico: erickgastelum@uas.edu.mx

** Doctor en Ciencias de la Educación. Profesor de tiempo completo ATC en la Universidad Autónoma de Chihuahua. Actualmente se desempeña como Coordinador del Doctorado en Ciencias de la Cultura Física de la Facultad de Ciencias de la Cultura Física. Es Catedrático de las Facultades de Ciencias de la Cultura Física y de Filosofía y Letras. Correo electrónico: rmarin@uach.mx

*** Doctora en Ciencias de la Educación. Profesora de tiempo completo ATC en la Universidad Autónoma de Chihuahua. Es miembro del Sistema Nacional de Investigadores Nivel I. Se desempeña como catedrática en el posgrado (a nivel maestría y Doctorado) de la Facultad de Filosofía y Letras. Responsable y miembro del Cuerpo Académico Consolidado "Educación y Comunicación". Correo electrónico: iguzman@uach.mx

${ }_{* * * *}$ Doctor en Ciencias de la Actividad Física. Profesor e Investigador Titular de la Universidad Autónoma de Sinaloa. Línea de investigación: Prescripción del ejercicio físico para la salud. Profesor e Investigador de Tiempo Completo de la Facultad de Educación Física y Deporte. Correo electrónico: aldohdez80@hotmail.com
} 
Intervención en docentes para el desarrollo de competencias psicomotoras en niños a nivel preescolar

didácticas permitieron que los niños desarrollarán habilidades psicomotrices mediante actividades lúdicas, para los docentes, el proceso de intervención apoyado del método M-DECA, centrando en la concepción conceptual, metodológica y de aporte constructivo desarrolló una práctica incluyente y reflexiva. Las competencias psicomotoras adquiridas en los niños y las competencias docentes fueron beneficiosas para la socialización y desarrollo físico y de salud en los educandos, así como también para el docente la cual fortaleció y renovó su discurso y prácticas educativas, generando un dispositivo que puede ser empleado por otras instituciones.

Palabras Clave: Habilidades psicomotrices, competencias, intervención, formación profesional continua, aprendizaje colaborativo.

\section{Intervention in teachers for the development of psychomotor skills in preschool children}

\section{ABSTRACT}

The objective of the study was to describe the results of the application of the preschool education program supported by the M-DECA model in teachers for the development of psychomotor competencies in the formative field of physical development and health in children. The study had a qualitative approach, collaborative research, participant action research (PAR) method, 6 teachers and a group of 47 children from a preschool institution participated. The M-DECA model of Guzman, Marin and Inciarte (2014) was used, focusing on a guided practice of 7 activities, which were developed in five integral phases, each participant fulfilled several roles, to whom qualitative instruments and techniques systematized by ATLAS ti software were applied. The findings found after the application of the didactic sequence allowed the children to develop psychomotor skills through playful activities. For the teachers, the intervention process supported by the M-DECA method, focusing on the conceptual, methodological and constructive contribution conception, developed an inclusive and reflective practice. The psychomotor skills acquired in the children and the teaching skills were beneficial for the socialization and physical and health development of the students, as well as for the teachers, who strengthened and renewed their discourse and educational practices, generating a device that can be used by other institutions.

Keywords: Psychomotor skills, competencies, intervention, continuing professional development, collaborative learning.

\section{Introducción}

Actualmente, uno de los retos más importantes de la educación física (EF) es el logro de aprendizajes integrales de los estudiantes, que atienda, no solo el entrenamiento del cuerpo, sino que a través del movimiento se contribuya al descubrimiento de la corporeidad como manantial de sensaciones, asimismo, que el contacto físico y la participación activa constituyan las vivencias a partir de las cuales se construya el pensamiento y el crecimiento emocional dirigido por la acción formativa de los docentes (López Pastor et al., 2016). 
La formación de capacidades, habilidades y destrezas en la educación física ha tomado un gran auge en estos tiempos (Vernetta et al., 2020), la práctica de EF en los niños genera innumerables beneficios para los individuos que la realizan en las diferentes áreas de la conducta humana: afectiva, social, cognitivo y motriz. No obstante, para alcanzar dichas ventajas en los estudiantes de nivel preescolar, es menester implementar reformas integrales en este nivel educativo que estén alineados con el propósito de impulsar la formación integral del educando que favorezca, a partir de aprendizajes, el desarrollo de habilidades para enfrentar la vida (Gil-Madrona et al., 2008).

Estos aprendizajes y el desarrollo de habilidades psicomotrices se originan progresivamente entre los cuatro y diez años de edad, simultáneamente con la maduración del sistema nervioso (Goodway et al., 2011; Malina et al., 2004). Por esta razón, la etapa de preescolar es crucial para promover en los niños el progreso y el crecimiento de sus capacidades físicas y mentales, apoyado por factores del entorno y por un equipamiento amplio e interesante (Barnett et al., 2013), aunado a la formación y competencias de los docentes para planear y programar los procesos de enseñanza aprendizaje, que favorezcan la interacción con los niños.

Latinoamérica, se ha adherido a estos cambios de reformas curriculares en todos los niveles educativos, México no ha sido la excepción, planteando desde hace una década el desarrollo de nuevas políticas basadas en la praxis educativa y la gestión en la educación preescolar, por tal motivo, la Secretaría de Educación Pública (SEP) ha diseñado e implementado el Programa de Educación Preescolar (PEP), dentro de él se incorpora un campo formativo denominado "desarrollo físico y salud", el cual se centra en la promoción de la actividad física desde temprana edad, a fin de sensibilizar y fomentar hábitos de vida activos en los niños y niñas para la prevención de enfermedades y lograr el mantenimiento de estilos de vida saludables (SEP, 2011).

Por tanto, los niños y niñas deben contar con condiciones y capacidades básicas, que garanticen el afrontamiento de las actividades físicas con éxito (García-Jaén et al., 2018). Estas competencias psicomotrices son necesarias en la ejecución e interacción del quehacer que plantea el docente de EF, al no ser ejercidos o llevados a cabo dentro de la clase provoca descoordinación, desmotivación, incompetencia, hasta el punto de que la educación física no les resulte agradable a los estudiantes (Ruiz Pérez, 2000).

De esta manera, la figura del docente de EF representa una especial importancia, dado que debe ser capaz de realizar una serie de actividades teóricas y prácticas coherentes, que toman en consideración las características y particularidades de los estudiantes y el enfoque por competencias propuesto por el currículo del PEP; esta situación genera la necesidad de una aplicación consistente de dicho programa ante la existencia de docentes conservadores, faltos de interés y carentes de herramientas y estrategias de enseñanza adecuadas para desarrollar las competencias y habilidades requeridas para el sostenimiento de la actividad física como estilo de vida, promoción de la salud, recreación, disciplina, educación en valores e iniciación deportiva (López Pastor et al., 2016).

Estudios realizados evidencian que el empleo de nuevas estrategias o propuestas de intervención contribuyen a mejorar la enseñanza, por ello se consideró que en el marco del Modelo para el Desarrollo y Evaluación de Competencias Académicas (M-DECA) propuesto por Guzmán et al., (2014) y Marín et al., (2013), es posible investigar y conocer cómo los profesores de educación física de nivel preescolar realizan sus prácticas educativas mediante el desarrollo 
Intervención en docentes para el desarrollo de competencias psicomotoras en niños a nivel preescolar

de las competencias docentes de: trasposición didáctica, gestión de la progresión de las competencias, coordinación de la interacción pedagógica, aplicación de formas de comunicación educativa adecuadas, y valoración del logro de competencias), consideradas en dicho modelo.

En ese sentido, el propósito de nuestro estudio se orientó a la necesidad de generar estrategias didácticas, apoyadas en el M-DECA, para ser aplicadas en procesos de intervención áulica por docentes de EF, a fin de trabajar competencias psicomotoras en el campo formativo de desarrollo físico y salud del PEP, que permita investigar la pertinencia de las experiencias vividas, así como su efecto y valor que conduzcan a la validación de una propuesta viable para su empleo en las escuelas de preescolar en México.

En consecuencia, el objetivo de este artículo es describir los resultados de la aplicación del programa educación preescolar apoyado del modelo M-DECA en los docentes para el desarrollo de las competencias psicomotoras en el campo formativo de desarrollo físico y salud en los niños. Los cuales se sustentan en los procesos de planificación y diseño de la docencia mediante la construcción de dos dispositivos: uno de formación, que toma como base teórica y metodológica a la pedagogía de la integración (Roegiers, 2010), y a la teoría de las situaciones didácticas (Brousseau, 2007); el otro, de evaluación de competencias, que recoge los planteamiento de las bases de la evaluación auténtica (Monereo, 2009; Brown, 2015; Moria, Refnaldi y Zaim, 2017), que estima que si los educadores físicos adquieren y desarrollan dichos fundamentos y se adhieren a esas prácticas mejorarán su perfil y conocimientos en el desarrollo y evaluación por competencias en beneficio de su práctica docente y del campo del desarrollo físico y salud de los niños y niñas.

\section{Método}

La investigación realizada se ubica en la perspectiva cualitativa. Se empleó el método de investigación acción (IA), a fin de valorar el efecto de la aplicación, en un proceso de intervención áulica, de estrategias construidas con base en el M-DECA para el desarrollo de competencias psicomotoras en el campo formativo de desarrollo físico y salud en los niños del programa de educación preescolar.

La intervención áulica justifica la pertinencia del uso del método de IA bajo el enfoque de investigación-formación-acción (Suárez y Metzdorff, 2018), en la cual el docente juega los roles de sujeto y objeto en un proceso de docencia-investigación.

Esta denominación de investigación-formación-acción (IFA) se genera en la articulación de diferentes conceptos que concurren en la formación-investigación atendiendo a lo teórico-metodológico desde una óptica crítica y emancipatoria. Asimismo, se encuentran variantes en la investigación (auto) biográfica-narrativa (Bolívar y Domingo, 2019), en la perspectiva (auto) etnográfica (Alegre-Agís y Riccò, 2017) y en la investigación-acción docente (Anderson, Herr y Nihlen, 2007). De esta manera, la investigación sobre la práctica educativa coloca a los docentes en el papel de investigadores que los conduce a cuestionarse permanentemente sobre sus posiciones teóricas y praxis cotidiana.

En ese sentido, la investigación- formación-acción desarrolla tres funciones: de formación, que busca una práctica educativa transformada y transformadora, de acción en la transformación de dicha praxis, y de investigación en la producción de conocimiento y en su comprensión. Asimismo, puede afirmarse que la investigación- formación-acción no es 
investigación, ni formación, ni acción, como tampoco puede concebirse como la confluencia de los tres procesos, siendo más propio entenderla como un bucle recursivo y retroactivo de investigación<->formación<->acción.

\section{Participantes}

Se tuvo la participación de 6 docentes, (3) docentes en formación en diplomaturas en educación preescolar y EF, (2) licenciados en educación preescolar y (1) licenciado en EF (investigador), entre los 27 y 32 años de edad, los cuales fueron seleccionados por conveniencia debido a la disponibilidad y alcance del investigador. Todos los participantes conformaron el grupo de IFA de profesionales e investigadores que laboran en la Universidad Autónoma de Chihuahua, México y la Universidad del Zulia, Venezuela, dentro del marco de desarrollo de intercambios de formación profesional continua (Tabla 1). Asimismo, un grupo de 47 niños de tercer año de educación preescolar mexicano, asistentes al jardín de niños "Sewarare 1200", Chihuahua, donde reciben y forman a niños entre 4 a 6 años, comprende una matrícula de alrededor de 580 niños aproximadamente distribuidos en varias secciones. Los criterios específicos que se tomaron para la selección fueron: 6 años de edad próximos a iniciar el nivel educativo superior, ganas de participar y previa autorización de padres/madres 0 representantes, educandos del turno de la mañana. Esto permitió conformar un solo grupo a la hora de llevar a cabo la investigación. Todos los participantes manifestaron colaborar, por lo que les entregó un consentimiento informado donde validaron su participación y posterior intervención.

Tabla1. Participantes de la investigación

\begin{tabular}{|c|l|}
\hline Docentes & \multicolumn{1}{c|}{ Institución } \\
\hline 2 & $\begin{array}{l}\text { Universidad del Zulia: (1) docente en formación en diplomatura en } \\
\text { educación preescolar y (1) EF. }\end{array}$ \\
\hline 1 & $\begin{array}{l}\text { Universidad Autónoma de Chihuahua: docente en formación en } \\
\text { diplomatura en educación preescolar }\end{array}$ \\
\hline 3 & $\begin{array}{l}\text { Universidad Autónoma de Chihuahua: (2) licenciados en educación } \\
\text { preescolar y (1) licenciado en EF (investigador) }\end{array}$ \\
\hline Niños & \\
\hline 47 & Jardín de Niños "Sewarare 1200" No. 1142 Zona 074, Chihuahua \\
\hline
\end{tabular}

Fuente: Elaboración propia

\section{Diseño}

La investigación acción desde la perspectiva de la formación incorpora características participativas y colaborativas con un sentido de acción y reflexión sistemática desarrollada por investigadores que tienen intereses comunes. La orientación colaborativa busca hacer el mejor empleo de los talentos individuales, fomenta la trasferencia de conocimientos y capacidades, fuente de incentivo y creatividad, extiende las redes de investigación y beneficia la diseminación de las investigaciones (Cárdenas Londoño et al., 2020; Domínguez-Gaona et al., 2015).

Asimismo, el método de Investigación-Formación-Acción en su ejecución permitió la construcción de las secuencias didácticas y su aplicación dentro del aula con los niños. Las secuencias didácticas cubrieron siete tópicos o actividades (Tabla 2) las cuales se realizaron en 
Intervención en docentes para el desarrollo de competencias psicomotoras en niños a nivel preescolar

sesiones semanales, con una duración de una hora y media cada una, durante los meses de septiembre, octubre, noviembre y diciembre de 2019, para el desarrollo físico, coordinación, fuerza y equilibrio, así como también la manipulación de objetos de trabajo para resolver problemas y actividades diversas, que permitieron realizar un análisis reflexivo de la dinámica del aula.

Tabla 2. Secuencias didácticas

\begin{tabular}{|cl|l|}
\hline \multicolumn{2}{|c|}{ Actividades } & \multicolumn{1}{c|}{ Materiales } \\
\hline I. & La soga larga & Soga, cuerda o tabla. \\
\hline II. & Malabaristas & Borrador, revista o periódico, libro, pañuelo. \\
\hline III. & Jugamos con el cuerpo & Pañuelo, costal, cuerda, soga \\
\hline IV. & Control de movimientos & Borrador de pizarra \\
\hline V. & Agiliza deditos y manos & Masa, palote. \\
\hline VI. & Gallinas poniendo huevos & Globos de colores, pelotas. \\
\hline VIl. & ¡Traza, pinta y recorta! & Pincel para pintar, colores, tijeras para recortar. \\
\hline
\end{tabular}

Fuente: Elaboración propia

\section{Procedimiento}

Los seis integrantes del grupo de IFA asumieron la distribución de las acciones desarrolladas de acuerdo con el procedimiento metodológico que contempló cinco etapas (Figura 1).

Figura 1. Procedimiento de la investigación colaborativa

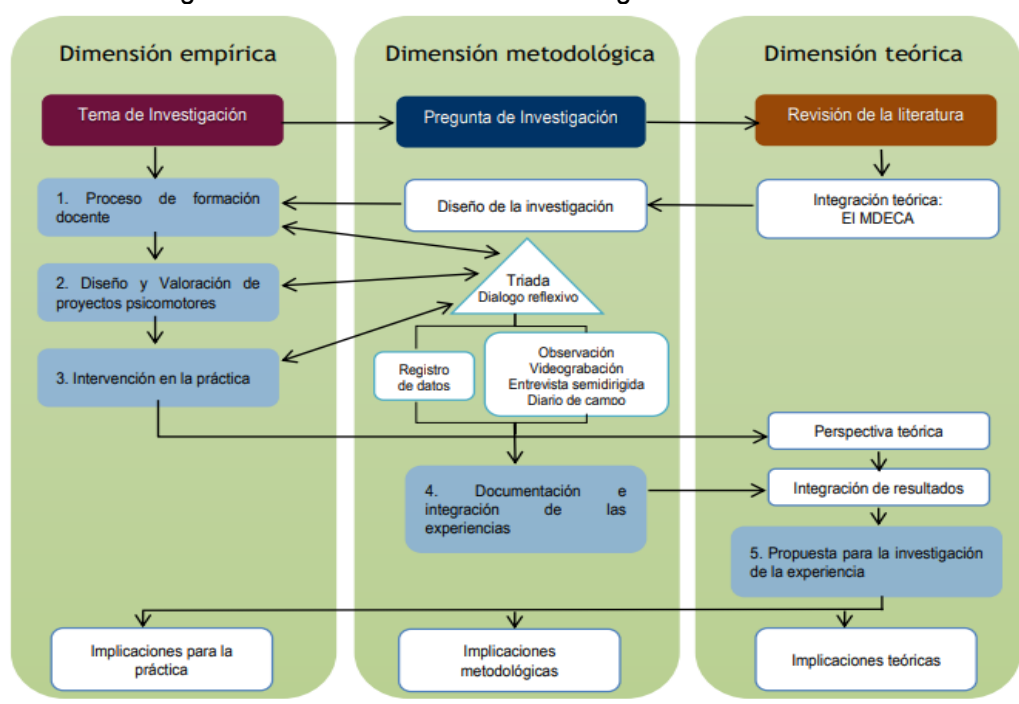

Fuente: Elaboración propia 
De esta manera, en el proceso de IFA se incorporaron los sustentos del M-DECA que incluyó, inicialmente, el proceso de formación docente, desarrollado en dos momentos: a) de práctica guiada, y b) de práctica autónoma (Guzmán et al., 2014). En el proceso formativo se cubrieron cinco módulos en dos etapas. En la primera etapa se impartieron los tres primeros módulos (práctica guiada) los cuales tuvieron como finalidad el estudio de la fundamentación teórica y metodológica para la construcción y validación de las secuencias didácticas (proyectos formativos psicomotores) para el desarrollo y evaluación de competencias. En la segunda etapa se desarrollaron los dos módulos finales (práctica autónoma), en ella se aplicaron los proyectos formativos psicomotores validados en un proceso de intervención en el aula, tomada como una articulación de docencia-investigación, asimismo, se dio la integración y valoración de los resultados de la experiencia.

En la primera etapa del proceso formativo, la construcción y validación de los proyectos formativos psicomotores se realizó con el apoyo de andamios cognitivos los cuales, en su estructura, consideran la tipología de actividades que vertebra el diseño de las secuencias didácticas que constan de tres categorías centrales: a) Descripción del objeto de estudio, b) Dispositivo de formación conformado por cuatro elementos: situación-problema, actividades de aprendizaje, evidencias de desempeño y recursos de apoyo, y c) Dispositivo de evaluación, que contiene estrategias, criterios e instrumentos de evaluación auténtica.

En la intervención áulica, correspondiente a la segunda etapa, el trabajo grupal se organizó en tríadas, las cuales se centraron en el asesoramiento y acompañamiento del proceso de aplicación de las secuencias prácticas para el desarrollo de competencias en el campo formativo de desarrollo físico y salud en el nivel preescolar, asimismo, en el desarrollo y la evaluación de competencias docentes de los profesores participantes en el proceso formativo, basados en la exhibición de sus producciones.

Dentro marco del desarrollo de intercambios de formación profesional continua los docentes de la Universidad del Zulia se encontraban en México realizando diplomaturas en educación preescolar y educación física durante los meses de la realización de la investigación por lo que el trabajo en triadas se asumió en diferentes momentos en tres diferentes roles: a) como presentador, quien preparó y aplicó la primera secuencia didáctica; b) como observador, quien observó, relató e informó lo que ocurrió antes, durante y después de la aplicación de la secuencia didáctica; y c) como facilitador, quien coordinó y distribuyó el trabajo, los roles se fueron cambiando durante todo el proceso formativo, el presentador en varias sesiones paso a ser observador, el observador paso a ser facilitador, y el facilitador paso a ser presentador, lo que contribuyó a la construcción, aplicación y valoración de los proyectos formativos psicomotores.

Es importante destacar que las intervenciones en el aula (práctica autónoma) se documentaron mediante registros, donde se emplearon instrumentos y técnicas cualitativas como la observación, diario de campo, rúbrica, diálogos reflexivos (trabajo de la tríada) y práctica videograbada.

El análisis e interpretación de los datos se realizó a través de la teoría fundamentada; esto facilitó la obtención de productos y conceptualizaciones teóricas "para entender el fenómeno estudiado, en un proceso ordenado y constante de comparación, análisis y codificación (De la Espriella y Gómez, 2020, p.127). Los seis docentes con el dominio de la práctica escolar le otorgaron fiabilidad en palabras y acciones a la investigación, lo que facilitó 
Intervención en docentes para el desarrollo de competencias psicomotoras en niños a nivel preescolar

la generación de categorías y sub-categorías como resultado de la intervención en los docentes para el desarrollo de competencias psicomotoras en niños a nivel preescolar. En la tabla siguiente se muestra las categorías emergidas en el estudio, mediante el apoyo del programa Atlas Ti 7.5.7 (San Martín, 2014).

Tabla 3. Categorías y Sub categorías creadas por el ATLAS.Ti.

\begin{tabular}{|l|l|}
\hline Grupo de documentos (sub categoría) & Código (Categoría) \\
\hline Sensaciones & Situaciones \\
Experiencias & \\
Acciones & \\
Cognitivo & \\
Socioafectivo & Actividades de aprendizaje \\
\hline Aprendizaje sugerente & \\
Debilidades & \\
Fortalezas & \\
Movimientos del cuerpo & \\
Juegos & \\
Estrategias. & Evidencia del desempeño \\
\hline Deseo por ir al preescolar & \\
Coordinación de movimientos & \\
Fomento de valores & \\
Desplazamientos & \\
Habilidades mentales. & \\
\hline
\end{tabular}

Fuente: Elaboración propia

\section{Interacciones sistemáticas de las experiencias educativas}

El ambiente pedagógico donde se desarrolló la investigación fue en las instalaciones del preescolar Jardín de Niños Sewarare ubicado en Chihuahua, con domicilio en la calle Río Uruguay S/N, perteneciente a la Zona Escolar clave 08EJN0043W, en el área de la educación pública, donde asisten un grupo de niños y niñas de 6 años de edad, correspondiente al tercero de preescolar. Se caracterizan por una actitud despierta y participativa, por lo que las clases son muchas veces interrumpidas por lo que requieren de la atención constante.

Los docentes a cargo del grupo son tres profesionales que están en formación en diplomaturas en educación preescolar y educación física, el resto de los tres docentes se encontraban en la realización de las actividades, donde cada uno realizó su planeación temática para llevar a cabo los contenidos a través de cuentos cortos que buscan el desarrollo de las capacidades mentales relacionadas con otras áreas del desarrollo físico y motor, todo ello para llamar la atención de los niños.

Por su parte, "la docente planteo varias preguntas a los niños y niñas, si les gustó el cuento, qué parte les gustó más y si algún personaje de la historia se parece a ustedes, por lo que respondieron que sí les gustó el cuento, que se parece a ellos porque quieren ser como alguno de los personajes, siéndole útil en su vida, para la toma de decisiones " 
Esto permitió reconocer a los docentes, que, si prestaron atención y si reflexionaron acerca de los personajes y la situación narrada, demostrando el cultivo de ciertas habilidades comunicativas en el proceso de enseñanza aprendizaje. Al principio la docente se mostró nerviosa, quizás porque estaba siendo grabada su práctica educativa y los niños pendientes de la cámara.

Seguidamente, se muestra que se retoma el liderazgo de la clase y los niños vuelven a la temática:

"se interacciona física y verbalmente, dramatizando la moraleja del cuento de la liebre y la tortuga"

\section{Docente 2}

Al mostrar dos contenidos importantes como la vergüenza que la liebre sintió ante la tortuga, y la humildad que se debe tener para tratar a las personas, ser sencillo, y reconocer nuestras equivocaciones, es parte del día a día, para ello hay que realizar actividades prácticas con elementos propios de la vida cotidiana de los niños.

Posteriormente, se señalaron las iniciales de las letras de los principales personajes del cuento, enseñando "letras del abecedario, desde la A hasta la Z"

\section{Docente 3}

De esta manera se buscó la integralidad de los niños, por medio de dos aspectos: primero el desarrollo de las capacidades mentales como la lógica, la memoria, y segundo la aplicación de los conocimientos de las letras para el desenvolvimiento frente a su entorno, la cual se verificó mostrando letras para su reconocimiento. De igual manera se observó el nerviosismo de la docente al ser filmada y los niños prestando atención a los agentes externos.

Cada actividad realizada requirió de los preescolares la atención, la memoria, comprensión y fluidez de sus pensamientos mediante el habla, puesto que al vivenciar la expresividad en la práctica se construye significados, desde una nueva manera de percibir y pensar para sentir, desarrollando así habilidades psicológicas (Del Valle-Núñez, 2015).

Las actividades del cuento, el dramatismo y el abecedario no solo reflejaron la intencionalidad de la actividad sino también el fomento de actitudes personales en los niños como la perseverancia, el respeto y la honestidad como elementos de equilibrio personal, social y de orden (Alberto del Salto Bello, 2015) destaca no solo la práctica educativa en sí, sino la vivencia que se trasmite en la relación docente-niño. Se observó la gran relevancia de la mediación de los docentes como agentes que proporcionan vivencias y experiencias, resultando uno de los adultos con más significancia en la vida del infante, dado que este puede potenciar, posibilitar o por el contario restringir el desarrollo de los niños que educa (Iglesias, 2019).

En este sentido, la práctica educativa reflejada por los docentes muestran su intento por involucrar diferentes elementos transversales y no sólo específicos de la enseñanza de los contenidos, corroborando que los docentes ofrecen espacios motivantes, actitudes tendientes a formar, presentando contenidos propuestos en los programas, sino también la adquisición de habilidades y actitudes propias del individuo que se ven proyectadas por su compromiso de formar integralmente a través de las experiencias (Longchamp-Gés, et al., 2018).

No obstante, hay que hacer hincapié en algunos elementos, como la innovación, reglas del acompañamiento y la evaluación (Barbosa-Chacón et al., 2015). En cuanto a la innovación 
Intervención en docentes para el desarrollo de competencias psicomotoras en niños a nivel preescolar

se observó vacíos en la práctica de la docente 2 aunque está reconoce las nuevas fuentes y metodologías, sin embargo, todavía sigue implementando sus prácticas educativas tradicionalistas. Contrariando lo planteado por Manrique Orozco y Gallegos Henao (2013), al referirse que en esta etapa se requiere de materiales pedagógicos y prácticas educativas que promueva el crecimiento de las competencias que estimulen la ejecución de las habilidades y mejoras del desempeño.

Los docentes acotaron que a medida que pasa el tiempo se requiere de nuevos saberes para no quedarse con lo que en algún momento aprendieron, por lo que es necesario una mejora en su educación continua, destacando de alguna forma lo señalado por Vergara (2015), que para mejorar la calidad de los aprendizajes en los niños y niñas, el docente debe renovar, diseñar, planificar, emplear recursos didácticos y evaluar los desempeños de sus estudiantes y la de ellos mismos, de manera tal que le permita cambiar prácticas educativas por otras que involucre la actualización de sus recursos e incorpore el trabajo colaborativo. Es decir, una reflexión permanente del quehacer pedagógico, fortaleciendo las competencias necesarias para su praxis educativa (Bohórquez-Casadiego et al., 2017).

Con respecto a las reglas de acompañamiento a través del grupo colaborativo el docente aprende con, de y por otros docentes mediante normas establecidas en los procesos de aprendizajes que potencia y a la vez facilita esa interacción e interdependencia provechosa y en el docente-docente y estudiantes-docentes actúan como co-aprendices (Fernández-Río y Méndez-Giménez, 2016). Estas reglas de acompañamiento conllevan a trabajar procesos de formación de docentes que lleven al desarrollo y evaluación de competencias docentes, transformando el binomio evaluación-formación en el bucle formación-evaluación (Marín et al., 2013), desarrollando competencias generales y específicas dentro del proceso formativo.

El disfrute por aprender desde la mirada de los niños quienes a través de actividades lúdicas se motivan, divierten y prestan atención, hacen silencio y manejan el material didáctico, ejecutando las actividades pedagógicas indicadas por sus docentes, los orienta a la adquisición de habilidades psicomotrices y a la vez el docente fortalece sus competencias establecidas en el M-DECA, desde la primera actividad propuesta en el programa que tiene como propósito mantener el control de movimientos que implica fuerza, velocidad y flexibilidad. El docente bajo sus distintos roles insistió en la práctica reiterada de los juegos, hasta que los niños las integrase a su repertorio conductual y las utilice de forma automática y habitual, seguidamente en la Tabla 4 , se muestra las actividades desarrolladas por los docentes y niños para el desarrollo de las competencias psicomotoras.

Tabla 4. Actividades para el desarrollo de las competencias psicomotoras

\begin{tabular}{|c|l|l|l|}
\hline Actividades & \multicolumn{1}{|c|}{ Acción docente } & \multicolumn{1}{|c|}{$\begin{array}{c}\text { Acción de los } \\
\text { niños }\end{array}$} & \multicolumn{1}{c|}{$\begin{array}{c}\text { Trabajo } \\
\text { colaborativo }\end{array}$} \\
\hline I & $\begin{array}{l}\text { Docente 3 (presentador): les indica a los } \\
\text { niños vamos a hacer una filita detrás de }\end{array}$ & $\begin{array}{l}\text { Siguieron las } \\
\text { indicaciones de }\end{array}$ & $\begin{array}{l}\text { Los docentes 2 y } \\
\text { 4: facilitadores }\end{array}$ \\
& $\begin{array}{c}\text { Yair todos, derechitos detrás de Rebeca, } \\
\text { los docentes. }\end{array}$ & Los docentes 5 y 6: \\
& pongan atención Anahí, detrás de & Realizando \\
& Cristina fórmate, Manuel fijate donde & circuito motriz \\
& estas, te estas saliendo de la fila, & sobre la soga o & \\
& Milagros intégrate, Listos vamos adores. & \\
& cuerda & \\
\hline
\end{tabular}




\begin{tabular}{|c|c|c|c|}
\hline & $\begin{array}{l}\text { avanzarle poquito aváncenle... tu hazte } \\
\text { hasta allá Brithany... necesitan espacio } \\
\text { para brincar, jahora si volteen para acá } \\
\text { Jesús! Fíjense vamos a hacer esto, } \\
\text { estamos de este lado de la soga y vamos } \\
\text { a brincar al otro lado... } \\
\text { Docente } 1 \text { (presentador): tú y tú. Vengan } \\
\text { los dos. Quiero que se vayan caminando } \\
\text { por la soga, arriba de la soga, caminando } \\
\text { los dos. Caminando, lento, poco a poco, } \\
\text { Luego indicó más rápido. Caminando } \\
\text { más rápido, sin caerse. Muy bien, } \\
\text { vengan. -Muy bien, ahora, ahora. Los } \\
\text { niños respondieron al unísono: Yo. Yo. } \\
\text { Yo. Yo. Yo. Todos van a pasar no se } \\
\text { preocupen, todos van a pasar. Hasta que } \\
\text { esté derechita la fila. ¡Muy bien! igual } \\
\text { quiero que pasen caminando todos, uno } \\
\text { tras otro, avancen. }\end{array}$ & $\begin{array}{l}\text { Caminar } \\
\text { trotar } \\
\text {. correr }\end{array}$ & $\begin{array}{l}\text { El grupo facilitó el } \\
\text { trabajo, grabando, } \\
\text { observando y } \\
\text { registrando } \\
\text { sucedido. }\end{array}$ \\
\hline II & $\begin{array}{l}\text { Docente } 5 \text { (presentador): indica vamos a } \\
\text { equilibrar nuestro cuerpo ¿cómo lo } \\
\text { podemos hacer? ¿Qué podemos hacer } \\
\text { para equilibrar nuestro cuerpo? ¿Qué es } \\
\text { equilibrio, diganme? ¿Quién sabe? } \\
\text { ¿Quién sabe que es equilibrar nuestro } \\
\text { cuerpo? ¿Alguien aquí lo dijo? Lo dijiste } \\
\text { tu victoria. Docente } 1 \text { (presentador) Si mi } \\
\text { amor, pero ahorita dijiste tú que un } \\
\text { equilibrista ¿qué es lo que hacía? Los } \\
\text { niños y niñas manifestaron: malabares, } \\
\text { aquellas personas que pasan por la } \\
\text { cuerda ¿qué también son los } \\
\text { malabaristas? Sí, los que caminan por la } \\
\text { cuerda ¿qué más? Niño: Los payasos } \\
\text { Maestra: y ¿qué hacen los payasos } \\
\text { malabaristas? Niño: Nos hacen reír los } \\
\text { payasos. } \\
\text { Docente } 6 \text { (presentador): primero antes } \\
\text { que todo les voy a platicar que los } \\
\text { malabaristas igual que ustedes mis niños, } \\
\text { fueron a la escuela, ellos también... ellos } \\
\text { utilizaron igual que ustedes, libretas, } \\
\text { borradores, también utilizaron, ... los } \\
\text { materiales que tenemos aquí, vamos a } \\
\text { ver que materiales usan los malabaristas } \\
\text { y que materiales usan los niños en la } \\
\text { escuela. A ver, vamos viendo estos } \\
\text { materiales ¿quién los utilizará? (les } \\
\text { enseña unas pelotas) ¿quién utiliza estos }\end{array}$ & $\begin{array}{l}\text { Siguieron las } \\
\text { indicaciones de } \\
\text { los docentes. } \\
\text { Realizando } \\
\text { circuito motriz } \\
\text { sobre la soga o } \\
\text { cuerda } \\
\text { Caminando con } \\
\text { las pelotas en las } \\
\text { manos } \\
\text { librotar con el borrador } \\
\text { de pizarra sobre } \\
\text { la cabeza uno } \\
\text { por uno. } \\
\text { Correr sobre la } \\
\text { cuerda }\end{array}$ & $\begin{array}{l}\text { El docente 2: } \\
\text { facilitador } \\
\text { Los docentes } 3 \text { y } 4 \text { : } \\
\text { observadores. } \\
\text { Cada uno facilitó el } \\
\text { trabajo, grabando, } \\
\text { observando y } \\
\text { registrando } \\
\text { sucedido. }\end{array}$ \\
\hline
\end{tabular}


Intervención en docentes para el desarrollo de competencias psicomotoras en niños a nivel preescolar

\begin{tabular}{|c|c|c|c|}
\hline & $\begin{array}{l}\text { materiales? Niña: los malabaristas } \\
\text { Maestra: los malabaristas ¿estos? (les } \\
\text { enseña un borrador de pizarrón) ¿aparte } \\
\text { de los maestros también ustedes lo } \\
\text { pueden utilizar no? Cuando pasan al } \\
\text { pizarrón ¿para qué utilizan esto? Niños: } \\
\text { para borrar, esto ¿lo utilizan ustedes? } \\
\text { (son libros) Niños: Sí Maestra }\end{array}$ & & \\
\hline III & $\begin{array}{l}\text { Docente } 1 \text { (presentador): les comunica a } \\
\text { sus niños que se coloquen en fila, van } \\
\text { agarrar el pañuelo cada uno por las } \\
\text { puntas como si fuera una soga, en pareja, } \\
\text { cada uno de un extremo, no se van a } \\
\text { soltar cuando llegue al extremo de la } \\
\text { cancha, sueltan el pañuelo y toman el } \\
\text { costalito, ¡Ángel y Karen! Vamos a llevar } \\
\text { estos pañuelos en forma de soga, igual } \\
\text { entre los dos, hasta allá y vamos a } \\
\text { regresar con el costal. Lo van a colocar } \\
\text { detrás de la línea, vamos a repetirlo, pero } \\
\text { ahora con el balón. Otra de la secuencia } \\
\text { fue con globos de colores en la cancha } \\
\text { los niños se emocionan ¡Muy bien! }\end{array}$ & $\begin{array}{l}\text { Siguieron las } \\
\text { indicaciones de } \\
\text { los docentes. } \\
\text { Realizando } \\
\text { circuito motriz } \\
\text { caminando } \\
\text { sosteniendo la } \\
\text { cuerda } \\
\text { (pañuelo). } \\
\text { Sosteniendo el } \\
\text { costal sin dejarlo } \\
\text { caer. }\end{array}$ & $\begin{array}{l}\text { Los docentes } 2,3 \text { y } \\
\text { 4: observadores } \\
\text { Los docentes } 5 \text { y } 6 \text { : } \\
\text { facilitadores. } \\
\text { Todos los docentes } \\
\text { facilitaron el } \\
\text { trabajo, grabando, } \\
\text { observando y } \\
\text { registrando } \\
\text { sucedido. } \\
\text { Conformación de } \\
\text { niños y niñas } \\
\text { trabajo en pareja. }\end{array}$ \\
\hline IV & $\begin{array}{l}\text { Docente } 3 \text { (presentador): puntualiza cada } \\
\text { borrador de pizarra, nos lo vamos a poner } \\
\text { en la cabeza, con cuidado, aquí en la } \\
\text { cabeza, a ver si es cierto que lo pueden } \\
\text { sostener en la cabeza caminando, vamos } \\
\text { a colocarnos derechitos (se van a ir } \\
\text { caminando al otro extremo de la cancha), } \\
\text { vamos a colocarnos aquí el borrador. } \\
\text { Luego fijense, yo lo voy hacer, nos vamos } \\
\text { a ir despacio caminando (se le cae el } \\
\text { borrador a la maestra) jay! Si se me cae, } \\
\text { fijense me tengo que detener volvérmelo } \\
\text { a colocar y no seguir avanzando hasta } \\
\text { que lo tenga bien colocado, sigo } \\
\text { avanzando, si se me cayó otra vez el } \\
\text { borrador, lo vuelvo a colocar, avanzo, } \\
\text { cuando llegue aquí fijense Evelyn me lo } \\
\text { va a quitar. Quítamelo Evelyn, se lo va a } \\
\text { colocar ella y avanza ¿sale? Ok mis } \\
\text { niños, empieza la actividad, hay que } \\
\text { realizarlo despacio, avancen (primero las } \\
\text { niñas y luego los niños) Se detienen, lo } \\
\text { colocan. No se vale sostenerlo con la } \\
\text { manita. }\end{array}$ & $\begin{array}{lr}\text { Siguieron las } \\
\text { indicaciones de } \\
\text { los docentes. } \\
\text { Realizando } \\
\text { circuito motriz de } \\
\text { extremo a } \\
\text { extremo de la } \\
\text { cancha } \\
\text { equilibrando el } \\
\text { cuerpo con el } \\
\text { borrador en la } \\
\text { cabeza. }\end{array}$ & $\begin{array}{l}\text { Los docentes } 1 \text { y } \\
\text { 2: facilitadores } \\
\text { Los docentes 4, } 5 \\
\text { y 6: observadores, } \\
\text { todos facilitaron el } \\
\text { trabajo, grabando, } \\
\text { observando y } \\
\text { registrando lo } \\
\text { sucedido. } \\
\text { Conformación de } \\
\text { grupos de niños y } \\
\text { niñas. } \\
\text { Trabajo de la } \\
\text { interdependencia } \\
\text { positiva para el } \\
\text { logro de metas. }\end{array}$ \\
\hline
\end{tabular}




\begin{tabular}{|c|c|c|c|}
\hline $\mathrm{V}$ & $\begin{array}{l}\text { docente } 4 \text { (presentador), hoy vamos a } \\
\text { trabajar algo bien divertido nada más que } \\
\text { tienen que poner mucha atención para } \\
\text { esta actividad que vamos a trabajar, } \\
\text { ¿sale? Ok. ¿quién de ustedes ha visto } \\
\text { hacer tortillas a mamá? ¿o conocen a un } \\
\text { panadero que haga bolillos? Algunos } \\
\text { niños dicen "yo", la docente pregunta } \\
\text { ¿Cómo los hace? ¿Qué movimientos } \\
\text { hace? Una de las niñas responde una } \\
\text { bolita y luego le hace así y así (le muestra } \\
\text { en la mesa como le hace el panadero) la } \\
\text { docente les indica estirar con un palote la } \\
\text { harina para adelante y para atrás, todos, } \\
\text { para adelante y para atrás. Despacio, } \\
\text { despacito, lento, lento, bien,... más lento } \\
\text { y luego ya las calienta, las cocina. }\end{array}$ & $\begin{array}{l}\text { Siguieron las } \\
\text { indicaciones de } \\
\text { los docentes. } \\
\text { Realizando } \\
\text { actividades } \\
\text { motrices con las } \\
\text { manos, } \\
\text { empleando } \\
\text { harina } \\
\text { apoyándose con } \\
\text { la mesa y un } \\
\text { rodillo de } \\
\text { madera. }\end{array}$ & $\begin{array}{l}\text { Los docentes } 1 \text { y } \\
\text { 2: Observadores } \\
\text { Los docentes } 3,5 \text { y } \\
6: \text { facilitadores, } \\
\text { todos facilitaron el } \\
\text { trabajo, grabando, } \\
\text { observando } \\
\text { registrando } \\
\text { sucedido. }\end{array}$ \\
\hline $\mathrm{Vl}$ & $\begin{array}{l}\text { docente } 5 \text { (presentador), vincula con los } \\
\text { ingredientes de la tortilla de la sesión } \\
\text { pasada, nombrando los ingredientes: } \\
\text { harina, huevos, margarina y leche. De allí } \\
\text { surge la pregunta ¿Qué animal pone } \\
\text { huevos? Todos los niños responden, las } \\
\text { gallinas. Quien sabe cómo ponen huevos } \\
\text { las gallinas... vamos a formar equipos de } \\
7 \text { niños, menciona el nombre de los niños } \\
\text { formando los equipos, hagan un círculo, } \\
\text { cuéntense } 1,2,3,4,5,6,7 \text { contando } \\
\text { niños, vénganse ustedes para acá Jaime, } \\
\text { haber hagan un círculo, agárrense de las } \\
\text { manos y hagan un círculo, haber yo les } \\
\text { ayudo, ahora Si....a la cuenta de } 3, \text { se van } \\
\text { a ir despacito, vamos a hacer de cuenta } \\
\text { que la gallina va a cargar el huevo. (no } \\
\text { entiendo, dice un niño), la docente } \\
\text { muestra, se coloca el globo entre las } \\
\text { piernas, lo tienen que llevar hasta allá } \\
\text { hasta donde está el pavimento y todos } \\
\text { juntos tienen que caminar sin que se les } \\
\text { caiga. ¿Ya vieron a sus compañeros } \\
\text { cómo están? el que tenga más huevos } \\
\text { gana. }\end{array}$ & $\begin{array}{l}\text { Siguieron las } \\
\text { indicaciones de } \\
\text { los docentes. } \\
\text { Realizando } \\
\text { circuito motriz } \\
\text { trasladando los } \\
\text { globos (huevo) } \\
\text { de un extremo de } \\
\text { la cancha a otro } \\
\text {. Caminando con } \\
\text { los globos en las } \\
\text { piernas } \\
\text {. Trotar con los } \\
\text { globos entre } \\
\text { piernas. } \\
\text { Correr con los } \\
\text { globos entre } \\
\text { piernas. }\end{array}$ & $\begin{array}{l}\text { Los docentes 1, } 2 \\
\text { y } 3 \text { : facilitador } \\
\text { Los docentes } 4 \text { y } \\
\text { 6: observadores } \\
\text { Todos los } \\
\text { docentes } \\
\text { facilitaron el } \\
\text { trabajo, grabando, } \\
\text { observando y } \\
\text { registrando lo } \\
\text { sucedido. } \\
\text { Conformación de } \\
\text { grupos de niños y } \\
\text { niñas. }\end{array}$ \\
\hline VII & $\begin{array}{l}\text { docente } 6 \text { (presentador), pregunta ¿Con } \\
\text { que parte del cuerpo se recogen los } \\
\text { huevos de gallina?, todos alegres } \\
\text { manifestaron: las manos... Pasaré por } \\
\text { sus mesas y les daré una hojita a cada } \\
\text { uno y en esa hojita van a tratar de dibujar } \\
\text { sus dos manitas, dibujan primero una, así }\end{array}$ & $\begin{array}{l}\text { Siguieron las } \\
\text { indicaciones de } \\
\text { los docentes. } \\
\text { Realizando } \\
\text { actividades de } \\
\text { motricidad, } \\
\text { trazando en } \\
\end{array}$ & $\begin{array}{l}\text { Los docentes } 1 \text { y } \\
\text { 3: observadores } \\
\text { Los docentes } 4 \text { y } 5 \text { : } \\
\text { facilitadores, todos } \\
\text { facilitaron el } \\
\text { trabajo, grabando, }\end{array}$ \\
\hline
\end{tabular}


Intervención en docentes para el desarrollo de competencias psicomotoras en niños a nivel preescolar

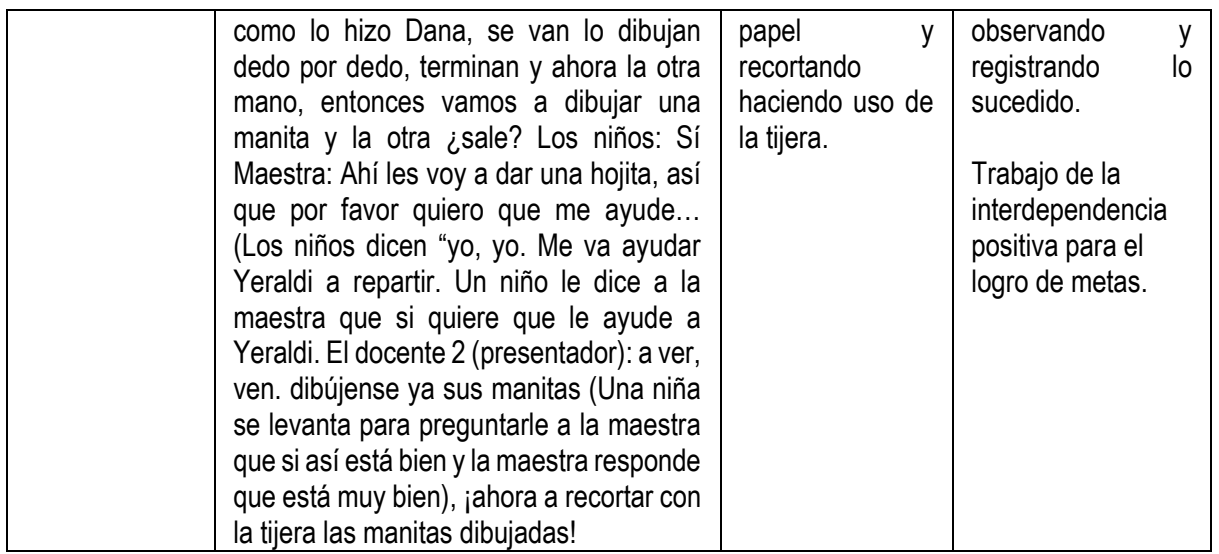

Una vez aplicadas las actividades de la secuencia didácticas desarrolladas por los docentes con base a los elementos en común en las entrevistas, emergieron las categorías que se analizaron para describir los resultados de la aplicación del programa educación preescolar apoyado del modelo M-DECA en los docentes para el desarrollo de las competencias psicomotoras en los niños y niñas de preescolar, reagrupando la información en tres categorías: situaciones, actividades de aprendizaje y evidencia de desempeño.

Situaciones

Las sesiones didácticas permitieron fortalecer las competencias psicomotrices en los educandos y las competencias docentes mediante el desarrollo de diferentes roles en cada sesión, todo el grupo de docentes colocaron en práctica sus habilidades, conocimientos, actitudes y recursos para solventar de manera favorable las diversas situaciones a las que se enfrentan día a día en su ejercicio profesional. En este sentido los docentes 2 y 3 señalaron:

Como se pudo observar las situaciones se dieron de manera natural por parte de los niños siguiendo instrucciones, ejercitando el cuerpo, y nosotros de forma ordenada y a conciencia aplicando y gestionando los contenidos en secuencia con los juegos para dejar un aprendizaje.

Las situaciones fueron emocionantes creando experiencias a través de las actividades físicas y motoras que estimularon y ayudaron a planear y generar otras con el objeto de mejorar el desarrollo psicomotor de los niños.

Se muestra que los educadores, consideran las intervenciones educativas como una posibilidad de vincular las acciones con los contenidos, el entorno del niño y la formación profesional del docente, lo que les permitió aplicarlas en diversas situaciones. Se logró por medio de metodologías divertidas, involucrando la lúdica, centrada en el intercambio y enriquecimiento mutuo cubriendo las necesidades de socialización, recreación y el desarrollo de movimientos corporales (Castro Pérez y Morales Ramírez, 2015).

Los docentes promueven el apoyo, diversificando las experiencias y estrategias de aprendizajes, que facilitan la integración y adquisición de capacidades integrales y específicas para desempeñarse con autonomía y autorregulación por medio de la planeación de su 
programa que sea el reflejo de la coherencia entre el discurso y su manera de actuar, generando desde las prácticas, ideas, habilidades y destrezas la integración del saber, con el ser y saber hacer, elementos principales para el éxito en los ambientes escolares (González Barajas, et al., 2010).

Debido a la significatividad de las acciones surgidas en ambientes reales, se pudo lograr que los docentes y los niños se apropiaran e integraran el conocimiento, producto de la construcción de las vivencias, sensaciones y emociones (Víquez-Zavala,2015), que son tomadas en cuenta para la elaboración del programa con un enfoque innovador complementando lo útil y beneficioso del ya existente.

Estas situaciones de aprendizaje, amplia a la educación física (EF), otorgándole beneficios y nuevas competencias para los estudiantes puesto que permiten potenciar las condiciones físicas básicas, la parte afectiva, cognitiva y relacional, tan relevante en el desarrollo integral de la personalidad del educando (Gil-Madrona et al, 2014).

Actividades de aprendizaje

Las actividades de aprendizaje planificadas por los docentes son fundamentales para el M-DECA, dado que el equipo se enfocó en el proceso de reflexión y estimación de las triadas efectuadas, donde los educadores 1 y 4 manifestaron que "nosotros no tenemos que hacer las actividades de aprendizaje y crear otro programa porque el programa ya está hecho y solamente hay que aplicarlo".

Sin embargo, se consideró significativo la postura del resto de los docentes quienes en planificadas situaciones fueron observadores, facilitadores y presentadores, al destacar el docente 6 "que algunas actividades de aprendizajes no están dentro del programa pero que pueden ser integradas logrando aprendizajes sugerentes y no sólo que se cumpla con el programa oficial". Sin saberlo, las docentes de alguna forma intervienen en la planeación de otro programa, tomando como bases las debilidades y fortalezas con la finalidad de enriquecer otra propuesta original.

De allí que los y las docentes, aunque no participen en el diseño curricular oficial, si planean sus actividades de aprendizajes arriesgándose mediante intervenciones de las secuencias educativas adaptarlas al contenido temático del programa diseñado para este nivel de preescolar, destacando la flexibilidad y autonomía del docente en la programación atendiendo las necesidades y requerimientos de los educandos. Esto es producto del nuevo rol que les toca asumir al momento de dar instrucción, debido a que actualmente los aprendices deben estar dotados de capacidades sociales, cognitivas y desarrollo físico, que les permita tener dominio y control del cuerpo y mente para la toma de decisiones y acciones (Gil-Madrona et al, 2014).

\section{Evidencia del desempeño}

La evidencia del desempeño dentro del modelo M-DECA se manifiesta en los docentes mediante el desarrollo de las competencias como producto de sus prácticas, el repensar de su actuación como sujeto evaluado y como participantes de su propia formación, el reconocer debilidades y fortalezas hacen mejorar las prácticas educativas. Estas evidencias reflejan una docencia renovada, capaz de incidir en la transformación de las situaciones de vida de quienes se forman. En atención a ello, el docente 5 manifestó "que nuestros estudiantes expresen que sus docentes son divertidos, cariñosos, pacientes y buenos enseñando, constatan que la 
Intervención en docentes para el desarrollo de competencias psicomotoras en niños a nivel preescolar

aplicación de estrategias lúdicas, deportivas fomentan actitudes y desarrollan habilidades que demuestran el progreso de sus aprendizajes"

Cada experiencia reveló la cercanía, cordialidad, respeto, confianza, convivencia y socialización, elementos que fortalecen la práctica educativa que es de mucha importancia para los educadores que los niños deseen ir a la escuela, porque encuentran en ella experiencias interesantes en su vida cotidiana, esa dinamización de la participación mediante actividades físicas con los niños prueban que los docentes fomentan estilos y hábitos de vida saludables beneficiosos para los niños (Castillo-Rodríguez et al, 2018)

Las actividades fueron muy fáciles y prácticas donde se logró que el niño adquiriera competencias motrices que es un elemento del rendimiento escolar (Becker et al., 2014; GarcíaMarín y Fernández-López, 2020), actividades que fueron tomadas en cuenta para proponer otro programa centrado en actividades psicomotrices que permitirá apreciar el proceso y los resultados que puede llegar a alcanzar los niños y docentes.

De esta manera, se plantea que los niños y niñas con buena competencia motriz se les posibilita una percepción positiva que los conduce a ser más activos y a mejorar su condición física, disminuyendo así inseguridades, así como padecer obesidad por falta de actividad física (García-Marín y Fernández-López, 2019). Desde el punto de vista de los observadores que paralelamente fungieron como facilitadores, presentadores y evaluadores, evidenciaron un desempeño de los docentes con una confianza que la metodología M-DECA genera, puesto que ella ayuda a reflexionar durante la acción para reajustar la práctica educativa, gestionando así los avances de los aprendizajes de sus estudiantes (Marín et al., 2013)

Los educadores participantes al poseer autoridad dentro de su espacio laboral y específicamente con sus estudiantes, argumentan que es necesaria para el aprovechamiento escolar develando la importancia de la intervención para hablar directamente y en forma individual cuando se enfrentan a la práctica educativa, no obstante, se marca la diferencia entre autoridad y autoritarismo haciendo hincapié en la influencia que tienen los educadores en el aprendizaje cuando muestra saber y tener competencia en su materia.

A partir de la implementación de las actividades lúdicas, se afianzó y mejoró habilidades psicomotrices que requirió el trabajo colaborativo, el valor social, la unión de todos para poder ganar. Asimismo, contribuyó a la aceptación de las normas sociales (Cobos Pino, 2011). Porque desde el ensayo, la práctica, y la experimentación de vivencias, se confirman y asumen cánones sociales en actividades organizadas o no organizadas (Gil-Madrona et al., 2014). Desde esta mirada se asume que los docentes participantes a pesar del miedo hacer observados, filmados y corregidos en la práctica, han reflejado experiencia, manejo y dominio de conocimientos en planificación, contenido de clase y dominio del grupo, descantado seguridad, manejo de emociones que han hecho que la intervención educativa fuese beneficiosa.

Estas actividades didácticas les permitieron a los niños de preescolar percibir la importancia de las partes del cuerpo, y como esta tiene utilidad para manipular y ejecutar las actividades en plena acción física, en el que el docente hace que el niño y niñas potencie su desarrollo motor, incorporando sesiones de motricidad en su planificación, haciendo uso de espacios adecuados atendiendo a las orientaciones del investigador y observadores especialistas en EF, hecho que coincide con Padial Ruz et al., (2019), al reconocer la importancia 
de la motricidad en la etapa preescolar, donde tiene que ser desarrollada curricular y extracurricularmente entre dos y tres horas a la semana.

De esta manera, el M-DECA es visto por los participantes desde una perspectiva pragmática, que ha fortalecido competencias generales y especializadas en un área poco trabajada en educación preescolar bajo concepciones del conocimiento útil y eficiente tanto para el educando como para el docente, existiendo un antes y un después, logrando el cumplimiento de tareas y responsabilidades, que permitió observar las fortalezas y áreas de oportunidad para mejorar las múltiples funciones que tiene el docente en el proceso de enseñanza aprendizaje (Romero-Martín y Arribas-Garralaga, 2020)

\section{Reflexiones}

La educación en la etapa infantil es la más gratificante e importante porque se trabaja con los niños quienes responden espontáneamente ante las situaciones de aprendizaje, es allí donde el docente observa con claridad su influencia y su huella. Desde allí, reconoce que su actuación e intervención de su práctica pedagógica como orientar y evaluar el progreso del niño, es tan vital para su propio desarrollo profesional.

Describir los resultados de la intervención docente tras la aplicación del programa educación preescolar apoyado del modelo M-DECA para el desarrollo de las competencias psicomotoras en el campo formativo de desarrollo físico y salud en los niños, fue realmente positivo, por la participación activa de los niños y niñas, logrando que por medio de la aplicación de secuencias didácticas propias del programa se ejercitará el cuerpo con el apoyo de actividades lúdicas vinculadas con su realidad, lo que permitió el aprendizaje y el desarrollo psicomotor de los educandos.

Así mismo, se adquirió competencias en los docentes participantes no solo renovaron su discurso, sino sus rutinas, la práctica a través del modelo M-DECA fue un proceso innovador, donde se mostró la observación, la evaluación autentica del proceso de adquisición y fortalecimiento de competencias en el docente que facilitó el diseño de otra propuesta diferente al programa gracias al diálogo reflexivo y al grupo colaborativo de investigadores especialistas en EF.

Sin duda alguna las intervenciones son de gran valor y producen grandes cambios, aunque al inicio fue intimidante para los docentes y estudiantes por las grabaciones. El desarrollo de las secuencias didácticas produjo varios efectos en los docentes que les permitieron internalizar y reflexionar acerca de la importancia de innovar y cambiar el quehacer educativo, por lo que se requiere una formación continua y permanente para hacerle frente a los nuevos retos del contexto escolar. Se adquirieron habilidades procedimentales, conceptuales y actitudinales, en un contexto particular que puede ser replicado en otros espacios; esta apropiación y asimilación facilitó la utilización funcional de contenidos y saberes diversos atendiendo la complejidad y exigencias de las situaciones reales en las que se aplican de manera eficaz y significativa en los niños.

En este sentido a raíz de las vivencias se pudo construir y generar un dispositivo refinado que conjuga un conjunto de actividades psicomotoras para desarrollar competencias en el campo formativo de desarrollo psicomotriz de los niños adaptados a los requerimientos del Programa de Educación Preescolar de México, que puede ser empleado en otras instituciones educativas. 
Intervención en docentes para el desarrollo de competencias psicomotoras en niños a nivel preescolar

\section{Referencias bibliográficas}

Alberto del Salto Bello, Max Walter. (2015). Educación en valores: propuesta de una estrategia. MEDISAN, 19(11).

Ecuador.

Extraído

de: http://medisan.sld.cu/index.php/san/article/view/533

Alegre-Agís, Elisa y Riccò Isabella. (2017). Contribuciones literarias, biográficas y autoetnográficas a la antropología médica en España: el caso catalán. Salud colectiva. Vol.13, núm.2. Argentina. (Pp. 279-293). Extraído de: http://www.redalyc.org/articulo.oa?id=73152115010

Anderson, Gary; Herr Kathryn y Nihlen, Ann. (2007). Studying your own School: An educators guide to practitioner action research. Corwin Press. 2da. Edición. EE.UU.

Barbosa-Chacón, Jorge Winston; Barbosa Herrera, Juan Carlos y Rodríguez Villabona, Margarita. (2015). Concepto, enfoque y justificación de la sistematización de experiencias educativas: Una mirada "desde" y "para" el contexto de la formación universitaria. Perfiles educativos, Vol. 37, núm. 149, México (Pp.130-149). Extraído de http://www.scielo.org.mx/scielo.php?script=sci_arttext\&pid=S0185-

$26982015000300008 \& \operatorname{lng}=e s \& \operatorname{lng}=e s$.

Barnett, Lisa; Hinkley, Trina; Okely, Anthony y Salmon, Jo. (2013). Child, family and environmental correlates of children's motor skill proficiency. Journal os Science and Medicine in Sport. Vol.16, núm, 4, Australia. (Pp. 332-336). doi: http://dx.doi.org/10.1016/j.jsams.2012.08.011

Becker, Derek; McClelland, Megan; Loprinzi, Paul y Trost, Stewart. (2014). Physical activity, selfregulation, and early academic achievement in preschool children. Early Education and Development, Vol. 25, núm. 1, Reino Unido. (Pp.56-70). https://doi.org/10.1080/10409289.2013.780505

Bohórquez-Casadiego, Mary Ramona; Durán-Chinchilla, Claudia Marcela y Rosado-Gómez, Alveiro Alonso. (2017). Valoración de las Competencias de los Docentes en El Contexto Universitario. Revista Ingenio. Vol. 13, núm 1, Colombia. (Pp. 147-57. https://doi.org/10.22463/2011642X.2143

Bolívar, Antonio y Domingo, Jesús. (2019). La investigación (auto)biográfica y narrativa en educación.Octaedro. España.

Brousseau, Guy. (2007). Iniciación al estudio de la teoría de las situaciones didácticas. Libros del Zorzal. Argentina.

Brown, Rally. (2015). La evaluación auténtica: El uso de la evaluación para ayudar a los estudiantes a aprender. Revista Electrónica de Investigación y Evaluación Educativa. Vol. 21, núm.2, España. (Pp. 1-10). http://dx.doi.org/10.7203/relieve.21.2.7674

Cárdenas Londoño, Rogelio; Mesa Velásquez, Gloria, y Restrepo Duque, Diego. (2020). Análisis de la producción científica y académica de los miembros de Redsicon: estudio de caso. Revista Visión Contable, Vol. 21. Colombia. (Pp.121-141). https://doi.org/10.24142/rvc.n21a6

Castillo-Rodríguez, Gema; Picazo Córdoba, Celestino y Gil-Madrona, Pedro. (2018). Revitalisation Recess as Conflict Resolution and Participation in Physical and Sports 
Activities. Revista Electrónica Educare, Vol. 22, núm. 2, Costa Rica. (Pp. 1-22). https://doi.org/10.15359/ree.22-2.14

Castro Pérez, Marianella y Morales Ramírez, María Esther. (2015). Classroom Environments That Promote Learning from the Perspective of School Children. Revista Electrónica Educare. Vol. 19, núm. 3, Costa Rica. (Pp. 1-32). https://doi.org/10.15359/ree.19-3.11

Cobos Pino, José. (2011). El juego motor en la escuela. Innovación y Experiencias Educativas. Vol. 40. España. (Pp. 1-8). Extraído de: https://archivos.csif.es/archivos/andalucia/ensenanza/revistas/csicsif/revista/pdf/Numer o 40/JOSE ANTONIO_COBOS_PINO 01.pdf

De la Espriella, Ricardo; y Gómez, Carlos. (2020). Teoría fundamentada. Revista Colombiana de Psiquiatría. Vol. 2. Colombia. (Pp.127-133). https://doi.org/10.1016/j.rcp.2018.08.002 Del Valle-Núñez, Andrea. (2015). Using Expressiveness and Creativity to Improve Human Relations: Reflections from the Teaching Practice. Revista Electrónica Educare, Vol. 19, núm. 1, Costa Rica. (Pp. 147-166). https://doi.org/10.15359/ree.19-1.9

Domínguez-Gaona, María del Rocío; Jitka Crhová y Molina-Landeros, Rosío del Carmen (2015). La investigación colaborativa: las creencias de los docentes de lenguas. Revista Iberoamericana de Educación Superior (RIES), Vol. vi, núm. 17, México (Pp.119-134). Extraído de: https://www.redalyc.org/pdf/2991/299141540007.pdf

Fernández-Río, Javier y Méndez-Giménez, Antonio. (2016). El aprendizaje cooperativo: Modelo pedagógico para Educación Física. RETOS. Nuevas Tendencias en Educación Física, Deporte y Recreación. Núm. 29. España (Pp.201-206). Extraído de: https://www.redalyc.org/articulo.oa?id=345743464040

García-Jaén, Miguel; Sellés-Pérez, Sergio; Cortell-Tormo, Juan; Férriz-Valero, Alberto., \& Cejuela, Roberto. (2018). Evaluación de los patrones de movimiento fundamentales en niños: comparación de género en escolares de Educación Primaria (Assessment of fundamental movement patterns in children: a gender comparison on Primary School students). Retos. Vol.34, https://doi.org/10.47197/retos.v0i34.65097

España.

(Pp.282-286).

García-Marín, Pablo y Fernández-López, Natalia. (2019). Asociación de la competencia en las habilidades motrices básicas con las actividades físico-deportivas extracurriculares y el índice de masa corporal en preescolares. Retos. Vol. 38, España. (Pp. 33-39). https://doi.org/10.47197/retos.v38i38.71896

Gil-Madrona, Pedro; Cachón-Zagalaz, Javier; Díaz-Suárez, Arturo; Valdivia-Moral, Pedro y Zagalaz-Sánchez, María Luisa. (2014). Las niñas también quieren jugar: La participación conjunta de niños y niñas en actividades físicas no organizadas en el contexto escolar. Movimento, Vol.20. núm. 1, Brasil (Pp.103-124). Extraído de: http://www.redalyc.org/articulo.oa?id=115329361006

Gil-Madrona, Pedro; Contreras-Jordán, Onofre R; Gómez-Villora, Sixto y Gómez-Barreto, Isabel. (2008). Justificación de la educación física en la educación infantil. Educación y Educadores, vol. 11, núm. 2, Colombia. (Pp. 159-177). Extraído de: http://www.redalyc.org/articulo.oa?id=83411211

González Barajas, Ma. Teresa; Kaplan Navarro, José César; Reyes Osua, Guadalupe y Reyes Osua, Mara Alejandra. (2010). La secuencia didáctica, herramienta pedagógica del 
Intervención en docentes para el desarrollo de competencias psicomotoras en niños a nivel preescolar

modelo educativo ENFACE Universidades. núm. 46, México (Pp. 27-33). Extraído de: http://www.redalyc.org/articulo.oa?id=37318636004

Goodway, Jacqueline; Ozmun, Jhon y Gallahue, David. (2011). Understanding motor development: infants, children, adolescents. Boston: NAVIGATE.

Guzmán, Isabel; Marín, Rigoberto, e Inciarte, Alicia. (2014). Innovar para transformar la docencia universitaria. Un modelo para la formación por competencias. Universidad del Zulia, Venezuela.

https://issuu.com/rigobertomarinuribe/docs/innovar_para_transformar_la_docenci

Iglesias, Greter Saura. (2019). La relación de la educadora con el niño de edades preescolares. Integración Académica en Psicología. Vol. 7 núm.19. Cuba. (Pp.1-8). Extraído de: https://integracion-academica.org/anteriores/29-volumen-7-numero-19-2019/226-larelacion-de-la-educadora-con-el-nino-de-edades-preescolares

Longchamp-Gés, Nuvía; Escalona-Vázquez; Irelis, Céspedes-Quiala, Alexis. (2018). La interdisciplinariedad: algunas consideraciones desde la carrera Licenciatura en Educación Preescolar. Maestro y Sociedad. Número especial. Cuba. (Pp. 178-185). Extraído de: https://maestroysociedad.uo.edu.cu/index.php/MyS/article/view/3905/3387

López Pastor, Víctor Manuel; Pérez-Brunicardi, Dario; Manrique, Juan Carlos y Monjas, Roberto. (2016). Los retos de la Educación Física en el Siglo XXI. Retos. Nuevas tendencias en Educación Física, Deporte y Recreación, Vol.29, España (Pp.182-187). https://doi.org/10.47197/retos.v0i29.42552

Malina, Robert; Bouchard, Claude y Bar-Or, Oded. (2004). Growth, Maturation and Physical Activity. Human Kinetics.EE.UU.

Manrique Orozco, Anyela Milena y Gallego Henao, Adriana María. (2013). El material didáctico para la construcción de aprendizajes significativos. Revista Colombiana de Ciencias Sociales. Vol. 4, núm. 1 Colombia. (Pp. 101-108. Extraído de: http://www.funlam.edu.co/revistas/index.php/RCCS/article/view/952

Marín, Rigoberto; Guzmán, Isabel; Márquez, Amelia y Peña, Manuel. (2013). la evaluación de competencias docentes en el modelo DECA: anclajes teóricos Formación Universitaria. Vol. 6, núm. 6, Chile (pp. 41-54). Extraído de: http://www.redalyc.org/articulo.oa?id=373534463005

Monereo, Carles. (2009). La autenticidad de la evaluación. Extraído de: https://www.researchgate.net/profile/Carles-Monereo/publication/257922429

Moria, Elva; Refnaldi, Refnaldi y Zaim, Max. (2017). Using Authentic Assessment to Better Facilitate Teaching and Learning: The Case for Students' Writing Assessment. Advances in Social Science, Education and Humanities Research (ASSEHR). Vol.148. The Netherlands (Pp. 333-337). Doi: https://doi.org/10.2991/icla-17.2018.57

Padial Ruz, Rosario; Ibáñez-Granados, Delia; Fernández Hervás, Marina; y Ubago-Jiménez, José Luis. (2019). Proyecto de baile flamenco: desarrollo motriz y emocional en educación infantil (Flamenco dance project: motor and emotional development in early childhood education). Retos. Vol. 35, España (Pp.396-401). https://doi.org/10.47197/retos.v0i35.63292 
Roegiers, Xavier. (2010). Pedagogía de la integración: competencias e integración de los conocimientos en la enseñanza. Coordinación Educativa y Cultural Centroamericana y AECI. Colección IDER. Costa Rica.

Romero-Martín; María Rosario y Arribas-Garralaga, Silvia. (2020). Aprendizaje cooperativo, expresión corporal y competencias docentes en actividad física Revista Iberoamericana de Ciencia de la Actividad Física y Deporte. Vol.9 núm.1 España. (Pp.133-14). http://dx.doi.org/10.24310/riccafd.2020.v9i1.8310

Ruiz Pérez, Luis Miguel. (2000). Aprender a ser incompetente en educación física: un enfoque psicosocial». Apunts. Educación física y deportes, Vol. 2, núm. 60, España. (Pp. 21-25), Extraído de: https://www.raco.cat/index.php/ApuntsEFD/article/view/306959

San Martín, Daniel. (2014). Teoría fundamentada y Atlas.ti: recursos metodológicos para la investigación educativa. Revista Electrónica de Investigación Educativa, Vol. 16. núm,1. México. (Pp. 104-122). Extraído de: http://www.redalyc.org/articulo.oa?id=15530561008 SEP. (2011). Programa de estudio 2011. Guía para la educadora. Educación básica preescolar. México: $\quad$ https://es.slideshare.net/Pilill/programa-de-estudio-2011-gua-para-laeducadora-educacin-bsica-preescolar

Suárez, Daniel y Metzdorff, Valeria. (2018). Narrar la experiencia educativa como formación. La documentación narrativa y el desarrollo profesional de los docentes. Revista de Educación Espacios en Blanco, núm. 28. Argentina. (Pp. 49-74). Extraído de https://ojs2.fch.unicen.edu.ar/ojs-3.1.0/index.php/espacios-en-blanco/article/view/78

Vergara, Ofelia. (2015). Formación Continua de los Docentes de la Educación Infantil: ¿cuál debe ser el debate? Educação \& Realidade, Vol.40, núm.4. Brasil. (P.p.973986). https://doi.org/10.1590/2175-623651523

Vernetta, María; De Orbe, Maria y Salas, Alicia. (2020). Práctica extraescolar del baloncesto y calidad de movimiento en chicas adolescentes. Revista lberoamericana de Ciencias de la Actividad Física y el Deporte. Vol.9 núm. 2 España. (Pp.75-93). https://doi.org/10.24310/riccafd.2020.v9i2.9080

Víquez-Zavala, Erika. (2015). Children's Role in Classroom Decision-Making Dynamics: Reflections from Paulo Freire's Pedagogy of Freedom. Revista Electrónica Educare, Vol. 19, núm 1, Costa Rica. (Pp.131-146). https://doi.org/10.15359/ree.19-1.8 